\title{
Evaluation of national surveillance methods for detection of Irish dairy herds infected with Mycobacterium avium ssp. paratuberculosis
}

\author{
E. S. G. Sergeant, ${ }^{1 *}$ C. G. McAloon, ${ }^{2}$ J. A. Tratalos, ${ }^{2,3}$ L. R. Citer, ${ }^{4}$ D. A. Graham, ${ }^{4}$ and S. J. More ${ }^{2,3}$ \\ ${ }_{1}^{1}$ Ausvet Pty Ltd., Canberra 2617, Australia \\ ${ }^{2}$ UCD School of Veterinary Medicine, University College Dublin, Belfield, Dublin D04 W6F6, Ireland \\ ${ }^{3}$ UCD Centre for Veterinary Epidemiology and Risk Analysis, University College Dublin, Belfield, Dublin D04 W6F6, Ireland \\ ${ }^{4}$ Animal Health Ireland, 4-5 The Archways, Carrick on Shannon, Co. Leitrim N41 WN27, Ireland
}

\section{ABSTRACT}

The aim of this study was to evaluate the utility and cost-effectiveness of a range of national surveillance methods for paratuberculosis in Irish dairy herds. We simulated alternative surveillance strategies applied to dairy cattle herds for the detection of Mycobacterium avium ssp. paratuberculosis (MAP)-infected herds (case-detection) or for estimation of confidence of herd freedom from infection (assurance testing). Strategies simulated included whole-herd milk or serum serology, serology on cull cows at slaughter, bulk milk tank serology, environmental testing, and pooled fecal testing. None of the strategies evaluated were ideal for widespread national case-detection surveillance. Herd testing with milk or serum ELISA or pooled fecal testing were the most effective methods currently available for detection of MAP-infected herds, with median herd sensitivity $>60 \%$ and $100 \%$ herd specificity, although they are relatively expensive for widespread use. Environmental sampling shows promise as an alternative, with median herd sensitivity of $69 \%$, but is also expensive unless samples can be pooled and requires further validation under Irish conditions. Bulk tank milk testing is the lowest cost option and may be useful for detecting high-prevalence herds but had median herd sensitivity $<10 \%$ and positive predictive value of $85 \%$. Cull cow sampling strategies were also lower cost but had median herd sensitivity $<40 \%$ and herd positive predictive values of $<50 \%$, resulting in an increased number of test-positive herds, each of which requires follow-up herd testing to clarify status. Possible false-positive herd testing results associated with prior tuberculosis testing also presented logistical issues for both cull cow and bulk milk testing. Whole-herd milk or serum ELISA testing are currently the preferred testing strategies to estimate confidence of herd freedom from MAP in

Received September 14, 2018

Accepted November 28, 2018.

*Corresponding author: evansergeant@gmail.com dairy herds due to the good technical performance and moderate cost of these strategies for individual herd testing. Cull cow serology and bulk tank milk sampling provide only minimal assurance value, with confidence of herd freedom increasing only minimally above the prior estimate. Different testing strategies should be considered when deciding on cost-effective approaches for case-detection compared with those used for building confidence of herd freedom (assurance testing) as part of a national program.

Key words: Johne's disease, cull cow serology, bulk milk testing, environmental testing, herd testing

\section{INTRODUCTION}

Bovine paratuberculosis, or Johne's disease (JD), is characterized by chronic granulomatous enteritis that manifests in clinically affected animals as a proteinlosing enteropathy, causing diarrhea, hypoproteinemia, emaciation, and eventually death (Sweeney et al., 2012). Adverse effects on animal productivity in terms of lower milk yield (McAloon et al., 2016b), higher cull rates (Hendrick et al., 2005), reduced value for culled animals (Richardson and More, 2009), and possible decreases in fertility (Johnson-Ifearulundu et al., 2000) are key drivers of the attempt to control the disease at farm level. In addition, research exists to suggest that the etiologic pathogen, Mycobacterium avium ssp. paratuberculosis (MAP), may pose a zoonotic risk (Liverani et al., 2014; McNees et al., 2015). Accordingly, many major dairy-producing countries have established JD control programs aimed at reducing the prevalence of the disease (Geraghty et al., 2014).

In Ireland, control of nonstatutory diseases such as JD is coordinated by Animal Health Ireland (AHI; More et al., 2011); AHI operated a pilot JD control program from November 2013 to December 2016 and has recently launched the voluntary Irish Johne's Control Program (IJCP). The new program has 4 key objectives: (1) to identify test negative herds and provide farmers with the tools and knowledge to increase their 
confidence of freedom over time; (2) to identify infected herds and provide farmers with the tools and knowledge to control the disease and reduce within-herd transmission; (3) to further underpin the quality of Irish dairy and beef produce in the international marketplace; and (4) to improve calf health and farm biosecurity in participating farms.

In the long term, the best use of available funds for the IJCP are subject to ongoing discussion. Several methods may be used for the detection of infected herds (in support of objective 2), including individual milk or serum serology, bulk tank milk serology, or testing of environmental samples or individual or pooled fecal samples by real-time PCR or culture. In Ireland, ongoing surveillance for national proof of freedom from bovine brucellosis is conducted by the Department of Agriculture, Food and the Marine (DAFM) through a cow monitoring survey (CMS). The CMS collects blood samples from approximately $70 \%$ of cull cows that enter DAFM-approved slaughter plants, presenting a potential cost-effective source of samples for MAP testing, with goals of either identifying MAP-infected herds (case-detection) or providing evidence of herd freedom from infection (assurance testing). The DAFMapproved slaughter plants process $88 \%$ of all Irish dairy cattle aged $24 \mathrm{mo}$ and above annually, with a further $3 \%$ being slaughtered through local authority-approved slaughter plants and the balance (9\%) disposed of through the knackery system.

The aim of our study was to evaluate the suitability of a range of national surveillance methods, both for case detection and for providing confidence of herd freedom from infection, in terms of herd sensitivity, positive and negative predictive values, overall cost, and utility, including any logistical factors associated with their use.

\section{MATERIALS AND METHODS}

\section{Model Overview}

A stochastic simulation model was implemented in the $\mathrm{R}$ software environment, version 3.3.3 ( $\mathrm{R}$ Core Team, 2017), with some calculations and outputs produced using the R packages RSurveillance (Sergeant, 2016), gplots (Warnes et al., 2016), ggplot2 (Wickham, 2009), and gridExtra (Auguie, 2016). The model simulated potential surveillance methods applied to dairy cattle herds for the detection of MAP infection (case detection) or for estimation of confidence of herd freedom from MAP. Herd size, numbers of animals slaughtered, and within-herd prevalence were estimated from existing data on Irish dairy herds. Model outputs included herd-level sensitivity, specificity, and positive and nega- tive predictive values for annual herd testing for each of the methods evaluated. Expected cost per herd tested, as well as number of herds tested and overall cost per 100 infected herds detected were also estimated. Model inputs were entered as probability distributions, to reflect uncertainty about their true values, and each scenario was simulated for 50,000 iterations, with key outputs summarized as medians and $95 \%$ prediction intervals.

\section{Surveillance Strategies}

The surveillance strategies simulated were based on a variety of testing methods, including herd-testing with individual milk or serum ELISA annually, bulk tank milk ELISA, cull cow sampling with serum ELISA, herd testing with pooled fecal sampling, and environmental sampling, as summarized in Table 1. For all animaltesting strategies, eligible animals for testing were those $\geq 2$ yr old to be consistent with testing requirements for the IJCP (AHI, 2017). For on-farm testing a single annual test event was assumed, whereas cull cow monitoring strategies testing was assumed to be accumulated over a 12-mo period.

For environmental (ENV) and pooled fecal (PFT) sampling, samples were assumed to be tested with either culture or PCR. For ELISA herd-testing strategies (strategies 1 and 2), any animals with positive ELISA results were assumed to be tested in series with an ancillary fecal test (fecal culture or PCR) to provide assumed specificity of 1 , as is currently the case for herd-assurance testing in the IJCP. For annual bulk milk-tank (BMT) ELISA and cull CMS, a test-positive herd is one that has one or more positive test results but for which the true MAP infection status is not known and further testing is required to confirm whether the herd is truly infected. For other strategies, a positive test result confirms MAP infection in the herd. Testing and associated costs for confirmation of infection in test-positive herds are not considered in the model for BMT and CMS strategies because any follow-up herd testing is voluntary and follow-up processes for these strategies are not defined in the IJCP.

A previous review of the relative performance of PCR and culture of fecal samples found that the 2 assays have comparable performance (Meyer et al., 2019), so for this analysis we assumed that the 2 tests were interchangeable.

Testing costs for each strategy were based on current or estimated costs for the various tests (Table 2). For individual (mELISA) and bulk milk ELISA, we assumed no additional collection costs because samples are already collected as part of existing programs and are accessible at no further cost. For serum ELISA 
Table 1. Summary description of 8 simulated national surveillance strategies for the detection of Mycobacterium avium ssp. paratuberculosis (MAP)-infected Irish dairy herds ${ }^{1}$

\begin{tabular}{lll}
\hline Strategy & Abbreviation & Description \\
\hline 1 & mELISA & $\begin{array}{l}\text { Annual herd testing with a single milk ELISA for lactating cows and serum ELISA for dry cows and } \\
\text { males. }\end{array}$ \\
2 & sELISA & $\begin{array}{l}\text { Annual serum ELISA testing of all adults in the herd. } \\
\text { Serum ELISA testing of } 100 \% \text { of cull cows slaughtered annually in Department of Agriculture, Food and }\end{array}$ \\
3 & CMS_100 & the Marine (DAFM)-approved slaughter plants. \\
4 & CMS_075 & Serum ELISA testing of $75 \%$ of cull cows slaughtered annually in DAFM-approved slaughter plants. \\
5 & CMS_050 & Serum ELISA testing of 50\% of cull cows slaughtered annually in DAFM-approved slaughter plants. \\
6 & BMT & Annual bulk milk tank ELISA testing. \\
7 & ENV & Annual environmental sampling of a set of 6 samples from specified locations in the farm environment. \\
8 & PFT & Annual pooled fecal testing, with a pool size of 5, of all eligible animals in the herd.
\end{tabular}

${ }^{1}$ Each scenario was considered a single herd-test event annually, except CMS strategies, which assumed testing accumulated over a 12-mo period.

(sELISA) on abattoir samples, we assumed a lower overall cost, relative to collection of samples on farm, due to economies of scale assuming large numbers of samples and use of existing staff and logistics currently employed for brucellosis screening. Pooled fecal sampling includes a small charge for pooling of samples at the laboratory. For laboratory costs for feces and environmental samples, we assumed either culture or PCR were used at a similar overall cost.

\section{Input Distributions}

Input distributions are summarized in Table 3 and described in detail below.

Individual Milk and Serum ELISA. The commercial ELISA kits most commonly used in Ireland to detect MAP infection in dairy herds are the Idexx (Idexx, Westbrook, ME) and IDVet kits (IDVet, Montpellier, France). As they are very similar, we did not distinguish between these kits when calculating the test sensitivity. Nielsen and Toft (2009) suggested an estimate of 0.15 for the sensitivity of serum ELISA for the target condition "infected." For this analysis, uncertainty around this estimate was incorporated by modeling the sensitivity of serum ELISA as a beta distribution. In line with previous literature, 0.15 was used as the mode of a beta distribution, with a 95th percentile set at 0.3 (Pozzato et al., 2011; More et al., 2013; McAloon et al., 2016a; Meyer et al., 2019). The sensitivity of individual milk ELISA relative to serum is estimated at 0.88 (van Weering et al., 2007); therefore, for this study, the sensitivity of individual milk ELISA was modeled using a beta distribution with a mode of 0.13 and a 95 th percentile of 0.26 . In both cases, test specificity was modeled with a mode of 0.99 and a 5 th percentile of 0.98 (Nielsen and Toft, 2009; Pozzato et al., 2011; McAloon et al., 2016a).

BTM ELISA. The sensitivity of bulk tank ELISA is related to the within-herd apparent prevalence (Nielsen and Toft, 2014), and estimates of bulk tank sensitivity are often reported relative to within-herd seroprevalence thresholds. Sensitivity estimates for different seroprevalence levels were extracted from van Weering et al. (2007) based on the commercial cutoff point of the test. However, these results revealed very small sample numbers present within some seroprevalence groups and similar estimates for all seroprevalence levels $<5 \%$. Therefore, the extracted results were pooled across 2 distinct bands: $<5 \%$ seroprevalence and $\geq 5 \%$ seroprevalence. Within each band, the number of herds detected $(i)$ and the number tested $(t)$ was used to fit a beta distribution, with the $\alpha$ parameter equal to $i+1$ and $\beta$ parameter equal to $t-i+1$ (Vose, 2000). The resulting beta distributions had means of 0.13 and 0.50

Table 2. Assumed testing costs $(€)$, including farm visits, sample collection, and laboratory costs, used for evaluation of 8 potential national surveillance strategies

\begin{tabular}{|c|c|c|c|}
\hline Test & $\begin{array}{l}\text { Visit } \\
\text { fee }\end{array}$ & Laboratory & $\begin{array}{c}\text { Sample } \\
\text { collection }\end{array}$ \\
\hline Milk ELISA (on farm) ${ }^{1}$ & 0 & 2.75 & - \\
\hline Serum ELISA (on farm) ${ }^{1}$ & 0 & 5.86 & - \\
\hline Serum ELISA (abattoir) ${ }^{1}$ & 0 & 2.15 & - \\
\hline Milk ELISA (bulk milk) $^{1}$ & 0 & 7 & - \\
\hline Individual feces (culture or PCR) & 40 & 40 & 3 \\
\hline Pooled feces (pool of 5 , culture or PCR) & 40 & 45 & 15 \\
\hline Environmental (set of 6) samples (culture or PCR) & 40 & 240 & 18 \\
\hline
\end{tabular}

${ }^{1}$ Includes collection cost. 
for herds with seroprevalences of $<5$ and $\geq 5 \%$, respectively. The specificity of BMT testing was reported as $100 \%$ in 110 certified MAP-free herds, modeled as a beta distribution with $\alpha$ and $\beta$ parameters of 111 and 1, respectively (van Weering et al., 2007).

Environmental Testing. The use of environmental testing to identify infected herds has been increasingly investigated in recent years. Overall, these studies vary according to the sampling method used (e.g., boot swabs, liquid manure, or samples from specified locations), the number of sites sampled, and the diagnostic test and test kit used for the sample analysis (Lombard et al., 2006; Raizman et al., 2006; Lavers et al., 2013; Wolf et al., 2016; Hahn et al., 2017). Lavers et al. (2013) used a standardized environmental sampling protocol of 6 farm locations (2 each in manure storage areas, cow concentration areas, and cow maternity or sick pens) and reported a sensitivity of 0.71 (95\% CI $=0.49-0.86)$ relative to herd infection status, as determined by repeated individual fecal testing (by real-time PCR or culture). The mean and lower $95 \%$ confidence interval of this result was used to fit the mode and 5th percentile of a beta distribution, which was used to model the sensitivity of environmental sampling and testing relative to whole-herd individual fecal testing. As for bulk milk testing, sensitivity of environmental sampling is correlated with within-herd infection prevalence (Tavornpanich et al., 2008; Donat et al., 2016). Although the data available were not adequate to specifically model this correlation, the distribution used is based on data from herds with a wide range of prevalence values. The specificity of environmental testing was assumed to be 1 .

Pooled Fecal Testing. Several studies were available reporting the sensitivity of pooled fecal testing relative to individual testing. These studies varied according to the number of samples pooled, stratification of the target condition (e.g., low, medium, and high shedding), and the analysis method used. For this analysis, data were extracted from studies investigating the sensitivity of pool sizes of 5 , as these represented the most commonly reported pool size. Estimates of the sensitivity of pooled fecal culture vary widely among studies depending on the methodology used and the level of shedding of MAP in feces. Wells et al. (2002) reported the sensitivity of pooled fecal culture as 0.43 , 0.88 , and 0.94 for low-, medium-, and high-shedding animals, respectively, giving a mean sensitivity of 0.75

Table 3. Summary of input probability distributions used in simulating 8 potential national surveillance strategies

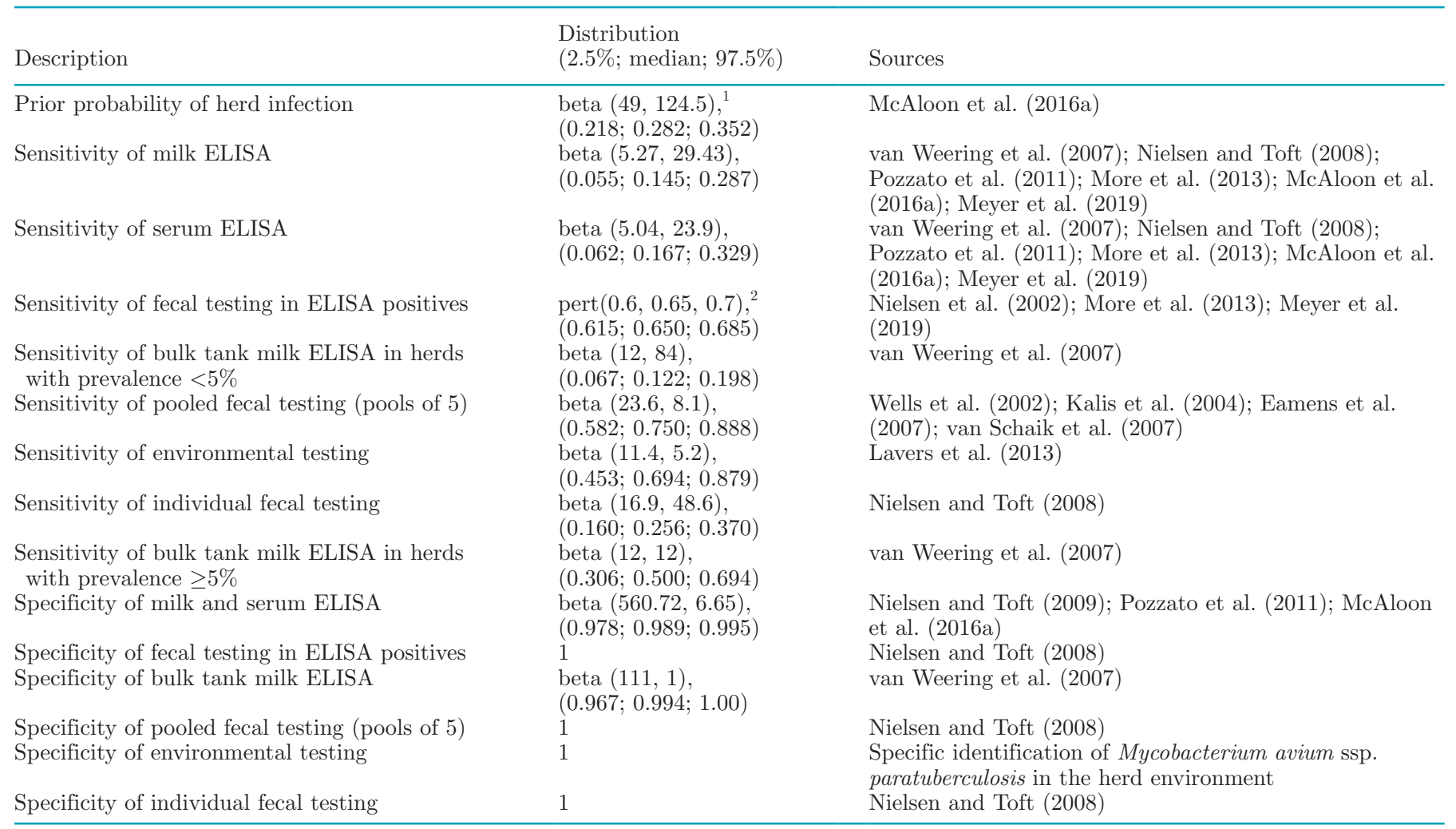

${ }^{1}$ Alpha and beta parameters for beta distributions.

${ }^{2}$ Parameters for pert distribution are minimum, most likely, and maximum. 
overall. Kalis et al. (2004) reported a sensitivity of 0.81, Eamens et al. (2007) reported a sensitivity of 1.00, and van Schaik et al. (2007) reported a sensitivity of 0.46. To accommodate the diversity of estimates, a beta distribution with a mode of 0.76 (the mean of the various estimates) and a 95th percentile of 0.86 was used to model uncertainty around this parameter $(\beta=23.6$, 8.1). The specificity of pooled fecal testing was assumed to be 1 .

Individual Fecal Testing. Herd testing with individual fecal testing was not specifically considered as a national surveillance strategy. However, herd-level sensitivity of individual testing was calculated as a reference point for the sensitivity of environmental sampling. Nielsen and Toft (2009) suggested a point estimate for the sensitivity of individual fecal testing of 0.25 for the target condition "infected." This value was used as the mode of a beta distribution with a 95th percentile of 0.35 .

As in previous studies, the sensitivity of individual fecal culture for confirmation of infection in ELISApositive animals was modeled as a PERT (0.6, 0.65, 0.7) distribution (Nielsen et al., 2002; More et al., 2013; Meyer et al., 2019). The specificity of fecal culture was assumed to be 1 .

Herd Data. Animal population and slaughter data from DAFM's Animal Identification and Movement database, for the period from July 1, 2015, to June 30,2016 , were extracted and cleaned as previously described (Meyer et al., 2019). All females $\geq 2$ yr of age and slaughtered at DAFM-approved slaughter plants during the relevant period were identified to their herd of birth as being the most likely source of MAP infection. The population and slaughter data of these herds $(\mathrm{n}=14,130)$ were then used as probability distributions for herd size (animals $\geq 2$ yr old, by sex and lactation status) and the number of females born in each herd that were slaughtered during the period of interest at DAFM-approved slaughter plants and were $\geq 2$ yr of age at the time of slaughter. The number of additional herds in which each animal resided before slaughter was also determined.

Within-Herd Prevalence. Within-herd true prevalence of MAP infection was estimated from screening data collected during the pilot phase of the program, as previously described (Meyer et al., 2019). Estimates from these data that were $>0$ were used as a probability distribution for within-herd true prevalence for evaluation of the ability of each strategy to detect infected herds. Separately, a fixed within-herd design prevalence of $5 \%$ was used for estimation of herd-level sensitivity and confidence of freedom for assurance testing in testnegative herds.
Prior Probability of Infection. The prior probability of herd infection with MAP, for estimation of positive and negative predictive values, was estimated from a published herd-level prevalence estimate of 0.28 , with $95 \%$ posterior probability interval of 0.23 to 0.34 (McAloon et al., 2016a). This parameter was estimated as a beta probability distribution with mode of 0.28 and 95th percentile of 0.34 , resulting in a $\beta(124.5,49)$ distribution. The expected number of infected herds $(4,900)$ in the population was estimated as $17,500 \times$ 0.28 , as there are approximately 17,500 commercial dairies in Ireland.

\section{Herd-Level Test Performance Measures}

Herd-Level Sensitivity and Specificity. Herdlevel sensitivities and specificities for each strategy were calculated as described below, depending on the individual strategy. Herd sensitivity ( $\mathbf{S e H})$ for the combined milk and serum ELISA strategy (strategy 1) was estimated using the formula

$$
\mathrm{SeH}=1-(1-S e)^{d},
$$

where $S e$ is the average test sensitivity for milk and serum ELISA, weighted by the numbers of lactating and nonlactating (dry cows or male) animals in the herd, multiplied by the sensitivity of individual fecal testing in ELISA-positive animals, and $d$ is the estimated number of infected animals in the herd, calculated as the estimated true prevalence for the herd, multiplied by herd size, rounded up to the next integer. Herd specificity ( $\mathbf{S p H})$ was assumed to be 1 due to follow up of ELISA-positive animals with an ancillary fecal test.

The SeH for the serum ELISA-only strategy (strategy 2) was estimated using formula 1 , where $S e$ is the test sensitivity for serum ELISA, multiplied by the sensitivity of individual fecal testing in ELISA-positive animals. The $\mathrm{SpH}$ was assumed to be 1 due to the follow up of ELISA-positive animals.

The $\mathrm{SeH}$ for the cow monitoring survey strategies (strategies 3-5) were estimated using the sep.freecalc function from the RSurveillance package in R, with sensitivity and specificity for the serum ELISA, estimated within-herd true prevalence, $\mathrm{N}=$ herd size and $\mathrm{n}=$ number of animals sampled, as arguments for the function (Cameron and Baldock, 1998; Sergeant, 2016). The $\mathrm{SpH}$ was estimated as $S p^{n}$, where $S p$ is the specificity of the serum ELISA and $n=$ number of animals sampled.

The $\mathrm{SeH}$ and $\mathrm{SpH}$ for bulk tank ELISA (strategy 6) were assumed to be the same as test sensitivity and specificity, depending on estimated within-herd true 
prevalence, whereas $\mathrm{SeH}$ and $\mathrm{SpH}$ for environmental sampling (strategy 7) were assumed to be the same as test sensitivity and specificity for this test.

The SeH for pooled fecal sampling (strategy 8) was estimated using formula 1, where sensitivity is the estimated pool-level sensitivity of pooled sampling and $d$ is the expected number of infected pools, estimated as the number of infected animals in the herd up to a maximum of the number of pools required to sample the whole herd ( $N$ divided by pool size, rounded up to the next integer). The $\mathrm{SpH}$ was estimated as $S p^{n}$, where $S p$ is the pool-level specificity of pooled sampling and $n$ is the number of pools required to sample the whole herd (Christensen and Gardner, 2000).

Herd-Level Positive and Negative Predictive Values. The $\mathrm{SeH}$ and $\mathrm{SpH}$ and prior probability of herd infection were used to estimate herd-level positive (HPPV) and negative (HNPV) predictive values using standard epidemiological formulae (Sergeant and Perkins, 2015).

Herd Sensitivity and Confidence of Freedom for Test-Negative Herds. The $\mathrm{SeH}$ for estimation of confidence of freedom in test-negative herds was estimated for all strategies using the same formulae, assuming follow up of positive samples with ancillary fecal tests where required and a test specificity of 1 . These estimates and an assumed $\mathrm{SpH}$ of 1 were then used in the standard formula for HNPV to estimate herd-level confidence of freedom, assuming a prior probability of infection of 0.28 (Martin et al., 2007; McAloon et al., 2016a).

\section{Measures of Cost-Effectiveness}

Testing Effort. The testing effort required for case detection of each strategy was estimated by calculating the number of herds required to be tested for 100 infected herds to have positive test results.

Testing Costs. The overall testing cost for each herd was calculated from testing costs in Table 2 and the number of animals sampled. For mELISA and sELISA strategies, costs include follow-up testing of 1 positive reactor with fecal culture or PCR in test-positive herds to confirm the presence of infection, including sample collection cost. Total costs for testing required for 100 infected herds to have positive test results was also calculated as a standardized measure for comparison of costs between strategies.

Testing Effectiveness. For each strategy and iteration, the simulated herd was determined to be either test-positive or not using a random binomial function with a sample size of 1 and the relevant herd sensitivity as the probability of detection. The number of herds detected, and case detection proportion, were then calcu- lated for each strategy as the total number and proportion of iterations in which infections were detected by that strategy. The expected numbers and proportions of test-positive herds identified and of true-positive and false-positive herds and false-negative herds, as well as the cost of testing, were then calculated for each strategy for a range of population coverage values.

\section{RESULTS}

\section{Herd Data}

A total of 14,130 herds met the inclusion criteria for our study. Separately, prevalence data were available for 1,523 herds where $\geq 20$ animals were tested and the resulting true prevalence estimate was less than 0.90 ; of these, 702 had an estimated prevalence greater than 0 and were included in the simulation. Herd size ranged from 20 to 1,189, with a median of 78 (Meyer et al., 2019). Estimated within-herd true prevalence in eligible herds ranged from 0.001 to 0.897 , with a median of 0.127 and $77 \%$ of simulated herds had a prevalence $\geq 5 \%$. A total of 291,721 females $\geq 2$ yr old born in eligible herds were disposed of through DAFM- or local authority-approved slaughter plants or knackeries during the period, with a mean and median of 21 and 18 animals, respectively, per herd, representing $23 \%$ of eligible females in the source herds. Of these, 238,718 were slaughtered at DAFM-approved slaughter plants and were included in our analysis. The number of adult cows originating from each herd and included in the analysis ranged from 0 to 214 , with a mean of 17 and median of 14 . There were $1.2 \%$ of herds that had 0 cows slaughtered, a further $2.7 \%$ had only 1 to 2 cows slaughtered, and $34 \%$ had $\leq 10$ cows slaughtered at DAFM-approved slaughter plants (Figure 1). About $32 \%$ of cows moved directly from their birth-herd to slaughter and a further $38 \%$ moved only once to a new herd, whereas about $12 \%$ moved to new herds 3 or more times during their lives, including some animals that moved up to 11 times to new herds. On average, cows included in our study resided in a mean of 2.15 herds during their lifetime.

\section{Strategy Performance for Case-Detection}

Herd Sensitivity and Specificity. Median SeH was lowest for BMT (0.08) and CMS strategies (0.21 to 0.37 , depending on strategy) and highest for PFT (0.83; Figure 2A). All estimates, except for ENV, had very wide prediction intervals due to a combination of uncertainty about test performance and the wide range of simulated herd sizes. The $\mathrm{SpH}$ was 1 for individual mELISA, sELISA, PFT, and ENV, as these strategies 


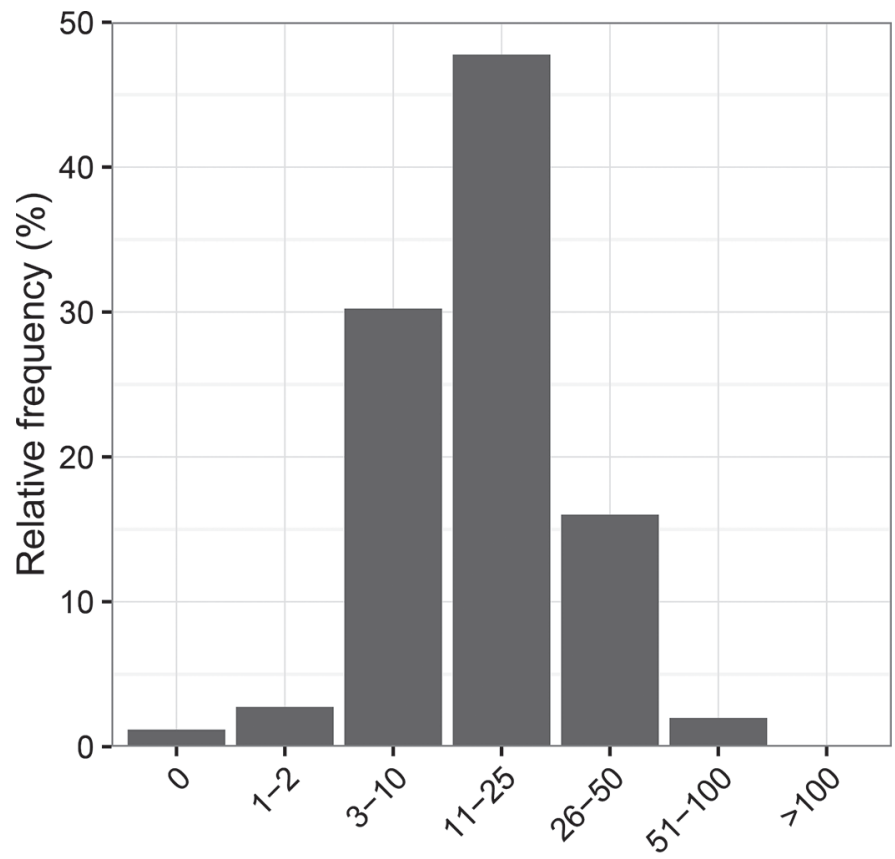

No. of females $>2$ yr old slaughtered per herd

Figure 1. Relative frequency distribution of numbers of adult females slaughtered at Department of Agriculture, Food and the Marine-approved slaughter plants per herd during the period from July 1, 2015, to June 30, 2016.

were assumed to have $100 \%$ specificity. For the other strategies, median $\mathrm{SpH}$ ranged from 0.86 for CMS_100 to 0.92 for CMS_050 and 0.99 for BMT (Figure 2B).

Herd-Level Positive and Negative Predictive Values. Herd-level positive predictive values were 1 for individual mELISA, sELISA, PFT, and ENV, as SpH was also $100 \%$ for these strategies, whereas median values for the remaining strategies ranged from 0.47 for CMS_100 to 0.5 for CMS_050 and 0.85 for BMT (Figure 2C). Median HNPV ranged from 0.74 for BMT to 0.94 for PFT (Figure 2D).

Testing Cost Per Herd. Median testing cost per herd was lowest for the BMT $(€ 2.75)$ and CMS (€15.05-30.10) strategies, and most expensive for PFT $(€ 1,000)$. Other strategies ranged from $€ 298$ for ENV to $€ 316$ for mELISA and €531 for sELISA (Figure 2E).

Herd Sensitivity Versus Positive Predictive Value. Consideration of the relationship between SeH and HPPV (Figure 3) shows that BMT and CMS strategies had both low sensitivity and only moderate positive predictive values at herd level, resulting in both a lower case detection rate and higher numbers of false-positive herds compared with other strategies that had higher values for both $\mathrm{SeH}$ and HPPV.

Case Detection Proportions. Overall, case detection proportions were similar to the respective median
SeH values. Assuming national surveillance of all herds with the various strategies, between 874 (BMT) and 4,308 (CMS_100) test-positive herds would be identified, of which between 740 (BMT) and 3,611 (PFT) would be truly infected (Table 4).

For BMT and CMS strategies, these numbers include between 15\% (134 herds for BMT) and 53\% (2,270 herds for CMS_100) false-positive herds that are indistinguishable from infected herds without additional onfarm testing. Total cost ranged from $€ 48,125$ for BMT to $€ 526,750$ for CMS_100 and up to $€ 17,500,000$ for PFT (Table 4). In contrast, testing of 6,000 herds based on mELISA, sELISA, PFT, or ENV would detect between 1,009 (mELISA) and 1,240 (PFT) infected herds at substantially higher cost than industry-wide surveillance with BMT or CMS, but with no false-positive herds.

Number of Herds Tested to Detect 100 Infected Herds. Between 428 (PFT) and 4,550 (BMT) herds are required to be tested for 100 infected herds to have positive test results, depending on the strategy chosen (Table 5). Imperfect specificity of BMT and CMS strategies results in additional uninfected herds providing false-positive results $(52.6 \%$ of all positive herds for CMS_100). These false-positive herds cannot be distinguished from truly infected herds that also test positive without further herd testing to clarify their status at significant additional cost. Conversely, imperfect $\mathrm{SeH}$ for all strategies means that some infected herds will remain undetected, particularly for BMT and CMS strategies, with between 174 (CMS_100) and 1,174 (BMT) false-negative herds.

\section{Strategy Performance for Herd Confidence of Freedom}

The pattern of median SeH and confidence of freedom estimates among strategies for a fixed withinherd design prevalence of $5 \%$ and assuming negative test results was generally similar to that using actual prevalence values, except that values for most strategies tended to be somewhat lower because of the lower design prevalence compared with estimated true prevalence in these herds (Figure 4). In general, the very low sensitivity of BMT and CMS strategies resulted in only minimal increase in confidence of freedom above the prior level of 0.72 compared with other strategies.

\section{DISCUSSION}

We believe ours is the first analysis comparing the performance and cost-effectiveness of several alternative surveillance strategies with the dual national surveillance objectives of detecting MAP-infected dairy 


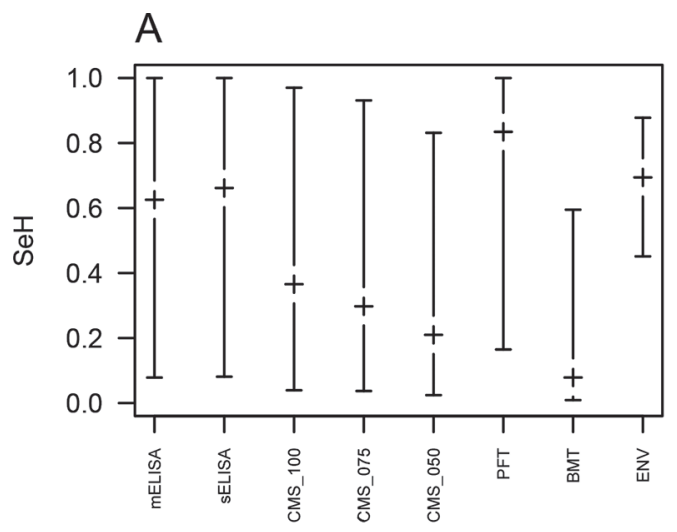

B
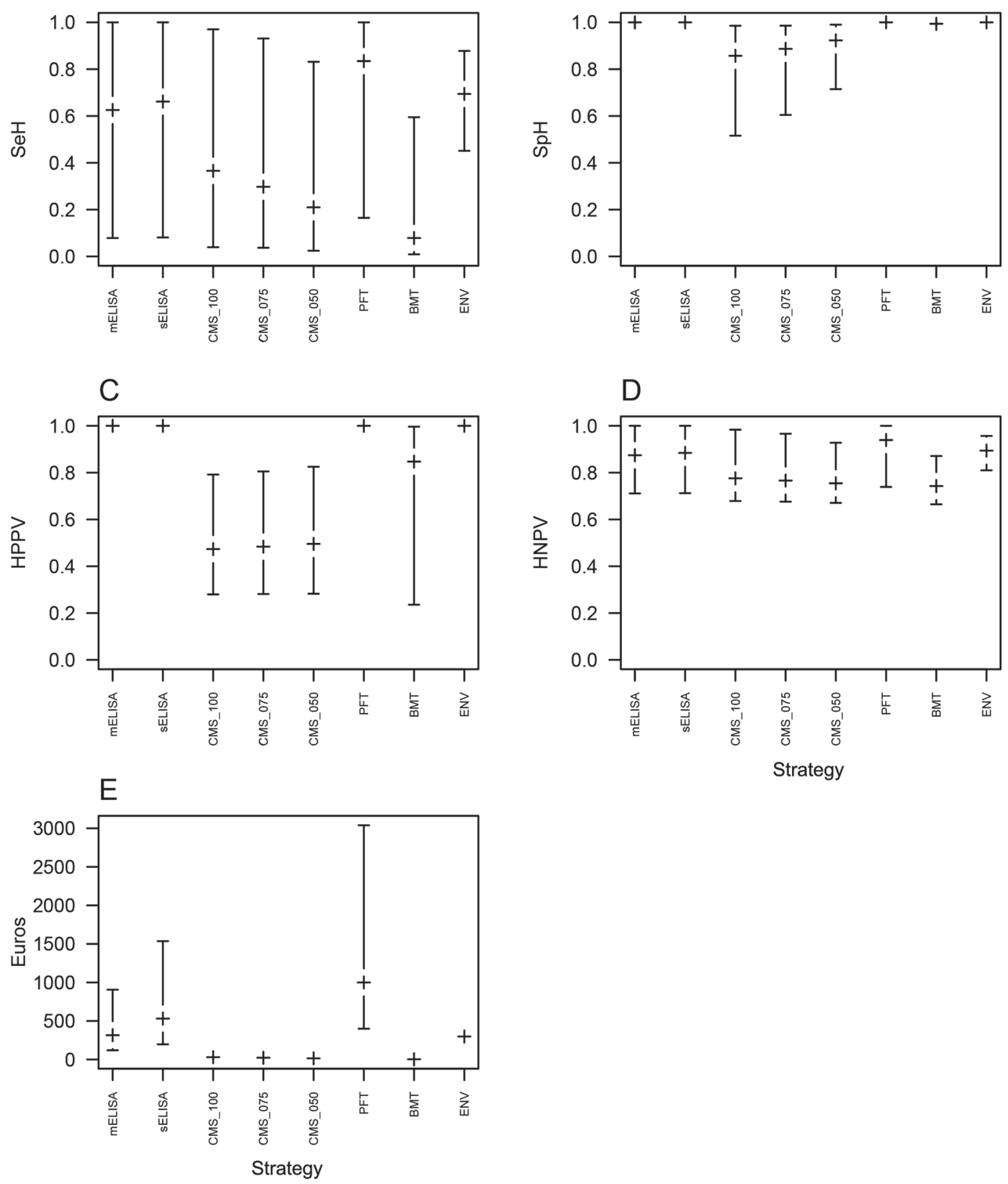

Figure 2. Median and 95\% prediction interval for herd-level sensitivity (SeH; A), herd specificity (SpH; B), positive predictive value (HPPV; $\mathrm{C})$, negative predictive value (HNPV; D), and testing cost $(€)$ per herd tested $(\mathrm{E})$ for 8 simulated national surveillance strategies (defined in Table 1).

herds and providing herd confidence of freedom in test-negative herds. One previous study was targeted at individual herd MAP-detection and cost-effectiveness, without considering industry-wide sampling or use of opportunistic sampling at slaughter plants such as has been undertaken in the current study (Tavornpanich et al., 2008). Our results will be used to inform future decision-making on national surveillance as part of Ire- land's national program. The analysis also provides a valuable resource for other countries considering the development of surveillance programs for MAP.

\section{Surveillance for Case Detection}

Based on our analysis, none of the strategies evaluated are ideal for widespread use for national case- 


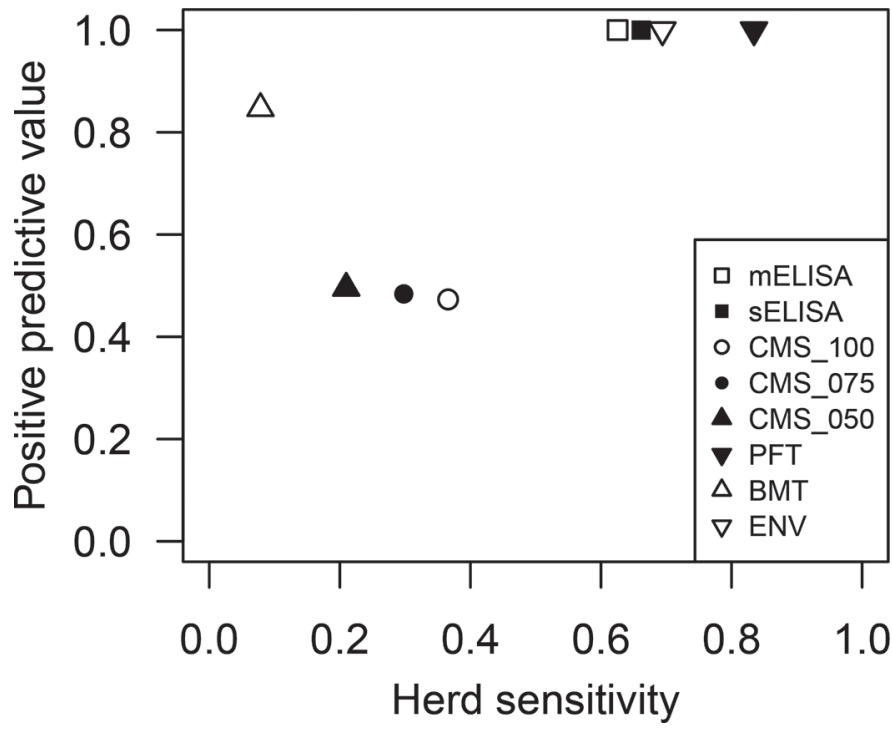

Figure 3. Scatter plot of median values for herd sensitivity $(\mathrm{SeH})$ and positive predictive value (HPPV) for 8 simulated national surveillance strategies (defined in Table 1).

detection surveillance. In general, lower-cost strategies such as BMT and CMS had lower values for both $\mathrm{SeH}$ and HPPV, resulting in more false-positive and false-negative herds compared with the higher-cost and better-performing strategies of mELISA, sELISA, PFT, or ENV.

Milk or Serum ELISA Whole-Herd Test. The 2 whole-herd ELISA testing strategies had similar and relatively good performance for case detection, with case detection proportions greater than $60 \%$ and positive predictive values of $100 \%$ due to follow-up of any seropositives with an ancillary fecal test. However, the requirement for whole herd testing and resulting high cost reduced the cost-effectiveness of these strategies as a tool for case detection during national surveillance, with costs exceeding $€ 5,000,000$ if applied nationally.

Cull Cow Monitoring Surveys. Whereas strategies based on abattoir sampling of cull cows were among the lower-cost strategies, the resulting herd sensitivities were considerably lower than more costly strategies and case detection proportions ranged from 27 to $42 \%$, depending on the assumed proportion of eligible cows tested, representing many infected herds being undetected at sampling. Further, these strategies have imperfect specificity, leading to false-positive results, requiring further follow-up of all test-positive cows with a herd test if the true herd status was to be determined. Median positive predictive values were around $50 \%$ for these strategies, so that about half of the herds with a positive serological result may in fact be uninfected.

Table 4. Expected total number of positive herds identified, number of these expected to be infected, percentage of all infected herds in the population tested positive, expected number and percentage of false-positive herds, and overall cost $(€)$ for national surveillance of all herds using 8 simulated national surveillance strategies compared with results for voluntary enrolment of $6,000,3,000$, or 2,000 herds in a test-based $\operatorname{program}^{1}$

\begin{tabular}{|c|c|c|c|c|c|c|}
\hline $\begin{array}{l}\text { Item }[\% \text { (no.) } \\
\text { of herds tested] }\end{array}$ & $\begin{array}{l}\text { Positive } \\
\text { herds }\end{array}$ & $\begin{array}{l}\text { Infected } \\
\text { herds }\end{array}$ & $\begin{array}{l}\% \text { of all infected } \\
\text { herds positive }\end{array}$ & $\begin{array}{c}\text { False-positive } \\
\text { herds }\end{array}$ & $\begin{array}{c}\% \text { False-positive } \\
\text { herds }\end{array}$ & $\begin{array}{c}\text { Total } \\
\text { cost }(€)\end{array}$ \\
\hline \multicolumn{7}{|c|}{$100 \%(17,500$ herds $)$} \\
\hline mELISA & 2,950 & 2,950 & 60.2 & 0 & 0 & $5,530,180$ \\
\hline CMS_100 & 4,308 & 2,038 & 41.6 & 2,270 & 52.7 & 526,750 \\
\hline CMS_075 & 3,637 & 1,759 & 35.9 & 1,878 & 51.6 & 395,062 \\
\hline CMS_050 & 2,691 & 1,333 & 27.2 & 1,358 & 50.5 & 263,375 \\
\hline \multicolumn{7}{|c|}{$34.3 \%$ (6,000 herds) } \\
\hline mELISA & 1,009 & 1,009 & 20.6 & 0 & 0 & $1,896,062$ \\
\hline sELISA & 1,039 & 1,039 & 21.2 & 0 & 0 & $3,185,290$ \\
\hline PFT & 1,240 & 1,240 & 25.3 & 0 & 0 & $6,000,000$ \\
\hline ENV & 1,161 & 1,161 & 23.7 & 0 & 0 & $1,788,000$ \\
\hline \multicolumn{7}{|c|}{$17.1 \%$ (3,000 herds) } \\
\hline mELISA & 338 & 338 & 6.9 & 0 & 0 & 632,021 \\
\hline sELISA & 348 & 348 & 7.1 & 0 & 0 & $1,061,763$ \\
\hline PFT & 412 & 412 & 8.4 & 0 & 0 & $2,000,000$ \\
\hline ENV & 387 & 387 & 7.9 & 0 & 0 & 596,000 \\
\hline
\end{tabular}

\footnotetext{
${ }^{1}$ Testing strategies as defined in Table 1.
} 

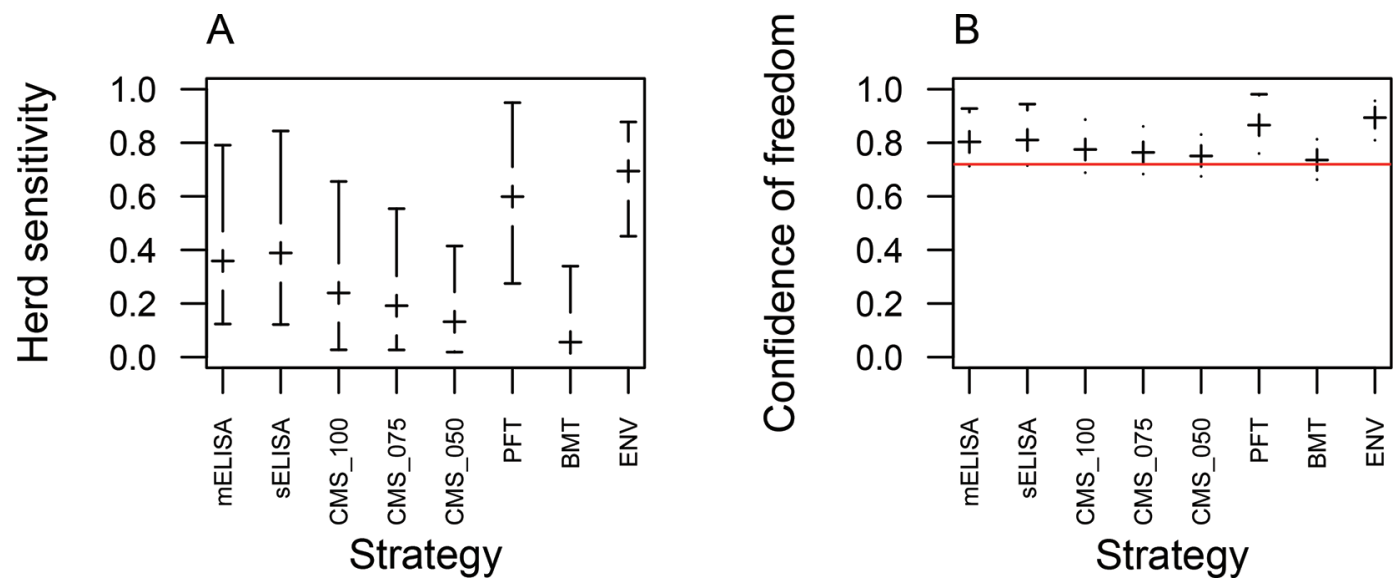

Figure 4. Median and 95\% prediction interval for herd sensitivity (A) and confidence of freedom (B) for 8 simulated national surveillance strategies (defined in Table 1), assuming a fixed within-herd design prevalence of $5 \%$. The horizontal line represents the prior confidence of freedom (0.72).

A comparison of strategies found that a national CMS strategy sampling all herds would potentially identify between 324 and 1,029 more infected herds than would testing of up to 6,000 herds using the mELISA strategy at between 14 and $28 \%$ of the cost (Table 4). However, CMS would also report high numbers of false-positive herds (up to 2,270 for CMS_100), which are indistinguishable from truly infected herds without additional testing, creating confusion and potentially discrediting the national surveillance program.

Cull cow testing strategies also present significant logistic issues to consider. Many dairy cows move, often multiple times, including to herds that may not operate as dairies. This would require careful consideration of program rules to determine which herd (or herds) should be considered at risk and notified of relevant testing and results, potentially at least doubling the number of herds to be tested compared with the actual number of test-positive herds detected, and significantly increasing overall costs for these strategies. Further, in a voluntary program, farmers may choose not to undertake any follow-up testing when notified of a positive test result because of cost issues or concern over implications of a positive confirmation, leaving these herds with no clear determination of their true infection status unless regulatory measures were introduced to require testing. Both undetected infected herds and test-positive herds that do not engage with the program would continue to contribute to ongoing spread of MAP to other herds, acting against the objectives of the program.

Currently, existing staff and procedures are already in place for sample collection for bovine brucellosis surveillance, so that sampling could use these existing resources at minimal additional cost. However, any changes to the existing brucellosis screening program or access to staff and logistics used for the program could significantly increase costs for these strategies.

The ELISA sensitivity for CMS strategies assumes an unbiased sample from the population. However, animals at slaughter are a biased sample and are likely to have a different age profile to the general population, so the actual sensitivity in this sample may also be

Table 5. Number of herd tests required, total testing cost $(€)$, total number of positive herds identified, and expected numbers and percentages of false-positive and false-negative herds for testing to identify 100 infected herds for 8 simulated national surveillance strategies

\begin{tabular}{|c|c|c|c|c|c|c|c|}
\hline Strategy & $\begin{array}{c}\text { Herds tested/ } \\
100 \text { infected } \\
\text { herds }\end{array}$ & $\begin{array}{l}\text { Cost/100 } \\
\text { infected } \\
\text { herds }(€)\end{array}$ & $\begin{array}{c}\text { Total } \\
\text { positive } \\
\text { herds identified }\end{array}$ & $\begin{array}{l}\text { False- } \\
\text { positive } \\
\text { herds }\end{array}$ & $\begin{array}{l}\% \text { False- } \\
\text { positive } \\
\text { herds }\end{array}$ & $\begin{array}{l}\text { False- } \\
\text { negative } \\
\text { herds }\end{array}$ & $\begin{array}{l}\% \text { False- } \\
\text { negative }\end{array}$ \\
\hline mELISA & 571 & 180,433 & 100 & 0 & 0 & 60 & 37.5 \\
\hline sELISA & 540 & 286,579 & 100 & 0 & 0 & 51 & 33.8 \\
\hline CMS_100 & 977 & 29,404 & 211 & 111 & 52.6 & 174 & 63.5 \\
\hline CMS_075 & 1,200 & 27,083 & 207 & 107 & 51.7 & 236 & 70.2 \\
\hline CMS_050 & 1,702 & 25,620 & 202 & 102 & 50.5 & 377 & 79 \\
\hline PFT & 428 & 428,024 & 100 & 0 & 0 & 20 & 16.7 \\
\hline BMT & 4,550 & 12,511 & 118 & 18 & 15.3 & 1,174 & 92.2 \\
\hline ENV & 514 & 153,311 & 100 & 0 & 0 & 44 & 30.6 \\
\hline
\end{tabular}

${ }^{1}$ Testing strategies as defined in Table 1. 
biased depending on the age profile and age-specific sensitivity values (Nielsen et al., 2013; Meyer et al., 2018). Nevertheless, any change in sensitivity is likely to be modest at best, and herd specificity and positive predictive value would remain poor and are unlikely to significantly change the relative performance of CMS compared with other strategies. Testing of adult male animals (bulls) at slaughter was not included in this analysis; however, because numbers are likely to be quite small for most herds compared with numbers of females, their inclusion would have only minimal effect on the estimates presented here and be unlikely to change the overall patterns of the results.

Bulk Tank Milk ELISA Testing. Bulk tank testing was the lowest cost option for case detection, requiring only the cost of a single test in each herd each year. As for the CMS strategies, the case detection proportion was low, at about $15 \%$. In contrast, the HPPV was substantially higher than CMS, at about $85 \%$, so that fewer false-positive herds would be expected. As for CMS testing, confirmatory herd testing to clarify the infection status of BMT-positive herds would be voluntary on the farmer's part and at significant cost to either the farmer or the program, although fewer herds would require follow-up.

One significant advantage of bulk milk testing is that the sensitivity of the assay is higher in higherprevalence herds, so it could be used for periodic herd screening to identify high-prevalence herds for followup advisory contact and assistance with developing an on-farm control plan for MAP. Finally, because bulk tank testing is inexpensive and can be undertaken on samples already collected for other purposes, it would be feasible to test herds on multiple occasions per year (subject to constraints of any effect of tuberculosis testing) for a relatively small investment, compared with some other strategies, further increasing the potential for case-detection.

Pooled Fecal Testing. Pooled fecal testing had the highest case detection proportion $(>70 \%)$ and $100 \%$ positive predictive value. However, the comparatively high cost (2-3 times that of milk or serum ELISA testing) is likely to limit its widespread use as part of a national surveillance program. One option to reduce costs for pooled fecal testing would be to increase pool size. A pool size of 10 was not specifically evaluated in this model, as robust estimates of pool-level sensitivity were not readily available for this pool size. However, some reduction in sensitivity and case-detection proportion would be expected, and the overall cost would remain similar to, or higher than, milk or serum ELISA testing.

Environmental Sampling. Environmental sampling performed nearly as well as pooled fecal testing, with a case detection proportion of $68 \%$ and $100 \%$ positive predictive value, but with a much lower cost, comparable to that for mELISA herd testing. An important consideration with environmental sampling is that this is a herd-level sample, with a fixed cost regardless of herd size. Thus, for larger herds (>about 108 animals) environmental sampling is lower cost than mELISA, whereas for smaller herds mELISA is cheaper. As for bulk milk testing, higher prevalence herds are more likely to produce a test positive result, although the relationship is not specifically simulated in this model.

Environmental sampling also relies on farmer cooperation for collection of samples; if significant numbers of farmers refuse to sample, the effectiveness of this strategy would be reduced.

In the future, it may be possible to substantially reduce the cost of environmental sampling and make it more cost-effective for all herd sizes by pooling of samples; for example, in 2 pools of 3 samples each, or a single pool of 6 samples. This is subject to further research to validate this approach.

Based on this analysis, environmental sampling has the potential to be the most cost-effective strategy for case detection, with high sensitivity and no falsepositive herds detected, particularly if cost can be reduced by pooling. However, these results are based on assumed values from work in Canadian dairies, so the applicability of these results in the Irish dairy industry must be considered. In particular, an appropriate set of environmental samples for collection in Irish dairies needs to be determined, standardized, and validated under Irish conditions, including potential pooling of samples and use of alternative sample matrices, before such a strategy could be fully implemented.

\section{Assurance Testing: Surveillance to Provide Confidence of Freedom}

Whole-Herd Milk or Serum ELISA Testing. Milk or serum ELISA are currently the preferred testing strategies for assurance testing, to estimate confidence of herd freedom from MAP in dairy herds, due to the good technical performance and moderate cost of these strategies. The results of this analysis are comparable to previous analyses and support the ongoing use of these tests for herd assurance (More et al., 2013; Meyer et al., 2019).

Pooled Fecal Testing. Pooled fecal testing would also be useful for assurance testing, except for the much higher costs compared with other options that also have reasonable herd sensitivity.

Environmental Sampling. Environmental sampling also has the potential to be a useful test for herd assurance, with similar performance to pooled fecal 
testing but at about one third the cost, subject to constraints already discussed.

Cull Cow Monitoring Survey and Bulk Milk Testing. In contrast, although having much lower costs, CMS and BMT strategies provide only minimal assurance value, as confidence of herd freedom increases only minimally above the prior estimate due to the generally small numbers of animals tested under CMS strategies, compared with a whole-herd test, and the generally poor sensitivity of BMT in low-prevalence herds. Additional research should be considered for further evaluation of the potential role of environmental sampling, bulk milk testing, and CMS strategies for herd assurance testing over time, either as initial screening tests or for maintenance of confidence of freedom once a target level has been achieved.

\section{Other Considerations}

Considerable uncertainty exists about some of the estimates presented in this analysis. This is partly due to the use of input probability distributions to allow for uncertainty about the true values of sensitivity and specificity estimates for the tests being modeled and the prior probability of a herd being infected. Variability in estimates is also introduced because of inherent variability in herd size and numbers of animals slaughtered in the data used. However, despite the uncertainty, the overall analysis is robust and provides a strong insight into the relative performance of the different strategies.

For the current analysis, the operating characteristics of the serological tests (individual sELISA, mELISA, and BTM ELISA) were as reported in the scientific literature. However, previous research has shown that intradermal skin testing for bovine tuberculosis reduces the specificity of individual milk and serum ELISA during the subsequent 2 to 3 mo (Kennedy et al., 2014). With CMS, which relies on the individual serum ELISA, animals slaughtered within 2 to 3 mo of tuberculin testing would need to be excluded from testing to avoid an increase in the number of false-positive results and, consequently, further reduction in HPPV for these strategies. However, this could substantially reduce the number of animals tested through CMS, noting that all cattle $>6$ wk of age are tested at least annually as part of the national tuberculosis eradication program (Good and Duignan, 2017), leading to a negative effect on the measured performance of CMS both for case detection and herd confidence of freedom. If the specificity of bulk milk ELISA was similarly affected, then a greater number of false-positive herds would also be expected. The bulk milk ELISA should be further evaluated under Irish conditions, and any effect of tuberculosis testing on specificity should be investigated before widespread use should be considered. Alternatively, milk testing should be timed to precede herd testing for tuberculosis, creating additional logistic issues for implementation. Similarly, serum or milk ELISA herd testing should be timed to precede or coincide with tuberculosis testing.

For this analysis, we assumed no differences in the performance of culture and PCR for detection of MAP in feces or environmental samples. This assumption was necessary because both assays are available for use in Ireland and a review of relevant literature found that most estimates of PCR performance were relative to fecal culture and either found no difference or a lower sensitivity, accompanied by several false positives that possibly were truly infected animals not detected by fecal culture (Meyer et al., 2019). Further, these tests were being applied as part of a herd test, so that issues such as passive ingestion of MAP and detection in feces of uninfected animals (Kralik et al., 2014) were still an indicator the herd was infected.

Cost estimates for this analysis were based on current costs and local knowledge, including extrapolation from existing cost structures. Although some of these costs might change once a program is implemented, any change is likely to be small and unlikely to affect the overall pattern of results, as overall costs are mainly influenced by the numbers of samples being tested and the large difference in costs between culture or PCR and serology.

\section{CONCLUSIONS}

Different national surveillance strategies should be considered when deciding on a cost-effective approach to case detection, as opposed to building confidence of herd freedom (assurance testing) as part of a national program. Herd testing with mELISA, sELISA, or PFT were the most effective methods for detection of MAPinfected herds, although they are relatively expensive. Environmental sampling shows promise as an alternative for national case detection if samples can be pooled for testing; without pooling, however, it is relatively expensive. Environmental sampling also requires further validation and determination of appropriate sampling sites and pooling rates under Irish conditions. Bulk milk tank testing is the lowest-cost option and may be useful, primarily for detection of higher-prevalence herds to allow targeting of advice to farmers to reduce within-herd prevalence and prevent spread to other herds, again subject to local evaluation and resolution of potential effects of tuberculosis testing on test specificity. Cull cow sampling strategies were also lower cost, but had generally lower herd sensitivity and very poor positive predictive values; this resulted in many 
false-negative herds and up to $50 \%$ false-positive herds, rendering the strategies unsuitable for widespread use for case detection without follow-up herd testing at additional cost. Whole-herd mELISA or sELISA testing are currently the preferred testing strategies to estimate confidence of herd freedom from MAP in dairy herds due to the good technical performance and moderate cost of these strategies. The CMS and BMT strategies provide only minimal assurance value, with confidence of freedom increasing only minimally above the prior estimate.

\section{ACKNOWLEDGMENTS}

The authors thank members of the Technical Working Group of the Irish Johne's Control Programme for helpful discussions during the preparation of this paper. The study was funded by the Irish Department of Agriculture, Food and the Marine, Dublin, Ireland.

\section{REFERENCES}

AHI. 2017. Irish Johne's Control Programme Interim Technical Manual. Animal Health Ireland, Carick-on-Sharron, Republic of Ireland.

Auguie, B. 2016. gridExtra: Miscellaneous Functions for "Grid" Graphics. 2.2.1 ed. R Foundation for Statistical Computing, Vienna, Austria. Accessed Jan. 13, 2019. https://cran.r-project.org/ web/packages/gridExtra/index.html.

Cameron, A. R., and F. C. Baldock. 1998. A new probability formula for surveys to substantiate freedom from disease. Prev. Vet. Med. $34: 1-17$.

Christensen, J., and I. A. Gardner. 2000. Herd-level interpretation of test results for epidemiologic studies of animal diseases. Prev. Vet. Med. 45:83-106.

Donat, K., N. Hahn, T. Eisenberg, K. Schlez, H. Kohler, W. Wolter, M. Rohde, R. Putzschel, U. Rosler, K. Failing, and P. M. Zschock. 2016. Within-herd prevalence thresholds for the detection of $M y$ cobacterium avium subspecies paratuberculosis-positive dairy herds using boot swabs and liquid manure samples. Epidemiol. Infect. 144:413-424.

Eamens, G. J., R. J. Whittington, M. J. Turner, S. L. Austin, S. A. Fell, and I. B. Marsh. 2007. Evaluation of radioetric faecal culture and direct PCR on pooled faeces for detection of Mycobacterium avium ssp. paratuberculosis in cattle. Vet. Microbiol. 125:22-35.

Geraghty, T., D. A. Graham, P. Mullowney, and S. J. More. 2014. A review of bovine Johne's disease control activities in 6 endemically infected countries. Prev. Vet. Med. 116:1-11.

Good, M., and A. Duignan. 2017. Veterinary Handbook for Herd Management in the bovine TB Eradication programme 2017. Department of Agriculture, Food and the Marine, Dublin, Ireland.

Hahn, N., K. Failing, T. Eisenberg, K. Schlez, P. M. Zschock, K. Donat, E. Einax, and H. Kohler. 2017. Evaluation of different diagnostic methods for the detection of Mycobacterium avium ssp. paratuberculosis in boot swabs and liquid manure samples. BMC Vet. Res. 13:259.

Hendrick, S. H., D. F. Kelton, K. E. Leslie, K. D. Lissemore, M. Archambault, and T. F. Duffield. 2005. Effect of paratuberculosis on culling, milk production, and milk quality in dairy herds. J. Am. Vet. Med. Assoc. 227:1302-1308.

Johnson-Ifearulundu, Y. J., J. B. Kaneene, D. J. Sprecher, J. C. Gardiner, and J. W. Lloyd. 2000. The effect of subclinical Mycobacterium paratuberculosis infection on days open in Michigan, USA, dairy cows. Prev. Vet. Med. 46:171-181.
Kalis, C. H. J., M. T. Collins, H. W. Barkema, and J. W. Hesselink. 2004. Certification of herds as free of Mycobacterium paratuberculosis infection: actual pooled faecal results versus certification model predictions. Prev. Vet. Med. 65:189-204.

Kennedy, A. E., A. T. Da Silva, N. Byrne, R. Govender, J. MacSharry, J. O'Mahony, and R. G. Sayers. 2014. The single intradermal cervical comparative test interferes with Johne's disease ELISA diagnostics. Front. Immunol. 5:564.

Kralik, P., R. Pribylova-Dziedzinska, A. Kralova, K. Kovarcik, and I. Slana. 2014. Evidence of passive faecal shedding of Mycobacterium avium ssp. paratuberculosis in a Limousin cattle herd. Vet. J. 201:91-94.

Lavers, C. J., S. L. McKenna, I. R. Dohoo, H. W. Barkema, and G. P. Keefe. 2013. Evaluation of environmental fecal culture for $M y-$ cobacterium avium subspecies paratuberculosis detection in dairy herds and association with apparent within-herd prevalence. Can. Vet. J. 54:1053-1060.

Liverani, E., E. Scaioli, C. Cardamone, P. Dal Monte, and A. Belluzzi. 2014. Mycobacterium avium subspecies paratuberculosis in the etiology of Crohn's disease, cause or epiphenomenon? World J. Gastroenterol. 20:13060-13070.

Lombard, J. E., B. A. Wagner, R. L. Smith, B. J. McCluskey, B. N. Harris, J. B. Payeur, F. B. Garry, and M. D. Salman. 2006. Evaluation of environmental sampling and culture to determine Mycobacterium avium subspecies paratuberculosis distribution and herd infection status on US dairy operations. J. Dairy Sci. 89:4163-4171.

Martin, P. A. J., A. R. Cameron, and M. Greiner. 2007. Demonstrating freedom from disease using multiple complex data sources 1: a new methodology based on scenario trees. Prev. Vet. Med. 79:71-97.

McAloon, C. G., M. L. Doherty, P. Whyte, L. O'Grady, S. J. More, L. L. M. Messam, M. Good, P. Mullowney, S. Strain, and M. J. Green. 2016a. Bayesian estimation of prevalence of paratuberculosis in dairy herds enrolled in a voluntary Johne's Disease Control Programme in Ireland. Prev. Vet. Med. 128:95-100.

McAloon, C. G., P. Whyte, S. J. More, M. J. Green, L. O'Grady, A Garcia, and M. L. Doherty. 2016b. The effect of paratuberculosis on milk yield-A systematic review and meta-analysis. J. Dairy Sci. 99:1449-1460.

McNees, A. L., D. Markesich, N. R. Zayyani, and D. Y. Graham. 2015. Mycobacterium paratuberculosis as a cause of Crohn's disease. Expert Rev. Gastroenterol. Hepatol. 9:1523-1534.

Meyer, A., K. Bond, S. Van Winden, M. Green, and J. Guitian. 2018. A probabilistic approach to the interpretation of milk antibody results for diagnosis of Johne's disease in dairy cattle. Prev. Vet. Med. 150:30-37.

Meyer, A., C. G. McAloon, J. A. Tratalos, S. J. More, L. R. Citer, D. A. Graham, and E. S. G. Sergeant. 2019. Modeling of alternative testing strategies to demonstrate freedom from Mycobacterium avium ssp. paratuberculosis infection in test-negative dairy herds in the Republic of Ireland. J. Dairy Sci. https://doi.org/10.3168/ jds.2018-14883.

More, S. J., M. L. Doherty, L. Downey, K. McKenzie, C. Devitt, and J. O'Flaherty. 2011. Animal Health Ireland: Providing national leadership and coordination of non-regulatory animal health issues in Ireland. Rev. Sci. Tech. 30:715-723.

More, S. J., E. S. G. Sergeant, S. Strain, W. Cashman, K. Kenny, and D. Graham. 2013. The effect of alternative testing strategies and bio-exclusion practices on Johne's disease risk in test-negative herds. J. Dairy Sci. 96:1581-1590.

Nielsen, S. S., C. Gronbaek, J. F. Agger, and H. Houe. 2002. Maximum-likelihood estimation of sensitivity and specificity of ELISAs and faecal culture for diagnosis of paratuberculosis. Prev. Vet. Med. 53:191-204.

Nielsen, S. S., and N. Toft. 2008. Ante mortem diagnosis of paratuberculosis: a review of accuracies of ELISA, interferon- $\gamma$ assay and faecal culture techniques. Vet. Microbiol. 129:217-235.

Nielsen, S. S., and N. Toft. 2009. A review of prevalences of paratuberculosis in farmed animals in Europe. Prev. Vet. Med. 88:1-14.

Nielsen, S. S., and N. Toft. 2014. Bulk tank milk ELISA for detection of antibodies to Mycobacterium avium ssp. paratuberculosis: Cor- 
relation between repeated tests and within-herd antibody-prevalence. Prev. Vet. Med. 113:96-102.

Nielsen, S. S., N. Toft, and H. Okura. 2013. Dynamics of specific anti-Mycobacterium avium ssp. paratuberculosis antibody response through age. PLoS One 8:e63009.

Pozzato, N., K. Capello, A. Comin, N. Toft, S. S. Nielsen, G. Vicenzoni, and N. Arrigoni. 2011. Prevalence of paratuberculosis infection in dairy cattle in Northern Italy. Prev. Vet. Med. 102:83-86.

R Core Team. 2017. R: A Language and Environment for Statistical Computing. R Foundation for Statistical Computing, Vienna, Austria.

Raizman, E. A., S. J. Wells, S. M. Godden, J. Fetrow, K. Friendshuh, and J. M. Oakes. 2006. Characterization of Minnesota dairy herds participating in a Johne's disease control program and evaluation of the program risk assessment tool. Prev. Vet. Med. 75:22-33.

Richardson, E., and S. More. 2009. Direct and indirect effects of Johne's disease on farm and animal productivity in an Irish dairy herd. Ir. Vet. J. 62:526-532.

Sergeant, E. 2016. RSurveillance: Design and Analysis of Disease Surveillance Activities. 0.2.0 ed. R Foundation for Statistical Computing, Vienna, Austria. Accessed Jan. 13, 2019. https://cran.r -project.org/web/packages/gridExtra/index.html.

Sergeant, E., and N. Perkins. 2015. Epidemiology for Field Veterinarians: An Introduction. CABI Wallingford, UK.

Sweeney, R. W., M. T. Collins, A. P. Koets, S. M. McGuirk, and A. J. Roussel. 2012. Paratuberculosis (Johne's disease) in cattle and other susceptible species. J. Vet. Intern. Med. 26:1239-1250.

Tavornpanich, S., C. A. Muñoz-Zanzi, S. J. Wells, E. A. Raizman, T. E. Carpenter, W. O. Johnson, and I. A. Gardner. 2008. Simulation model for evaluation of testing strategies for detection of paratuberculosis in Midwestern US dairy herds. Prev. Vet. Med. 83:65-82.

van Schaik, G., M. Pradenas, A. Mella, and J. Kruze. 2007. Diagnostic validity of pooled fecal samples and individual blood or faecal samples to determine the cow- and herd-status for Mycobacterium avium ssp. paratuberculosis. Prev. Vet. Med. 82:159-165.

van Weering, H., G. van Schaik, A. van der Meulen, M. Waal, P. Franken, and K. van Maanen. 2007. Diagnostic performance of the Pourquier ELISA for detection of antibodies against Mycobacterium avium ssp. paratuberculosis in individual milk and bulk milk samples of dairy herds. Vet. Microbiol. 125:49-58.

Vose, D. 2000. Risk Analysis-A Quantitative Guide. 2nd ed. John Wiley and Sons Ltd., Chichester, United Kingdom.

Warnes, G. R., B. Bolker, L. Bonebakker, R. Gentleman, W. H. A. Liaw, T. Lumley, M. Maechler, A. Magnusson, S. Moeller, M. Schwartz, and B. Venables. 2016. gplots: Various R Programming Tools for Plotting Data. 3.0.1 ed. R Foundation for Statistical Computing, Vienna, Austria. Accessed Jan. 13, 2019. https://cran .r-project.org/web/packages/gridExtra/index.html.

Wells, S. J., R. H. Whitlock, C. J. Lindeman, and T. Fyock. 2002 Evaluation of bacteriologic culture of pooled fecal samples for detection of Mycobacterium paratuberculosis. Am. J. Vet. Res. 63:1207-1211.

Wickham, H. 2009. Elegant graphics for data analysis. 2.2.1 ed. Springer-Verlag, New York, NY.

Wolf, R., H. Barkema, J. De Buck, and K. Orsel. 2016. Dairy farms testing positive for Mycobacterium avium ssp. paratuberculosis have poorer hygiene practices and are less cautious when purchasing cattle than test-negative herds. J. Dairy Sci. 99:4526-4536. 